\title{
T-DUALITY FOR PRINCIPAL TORUS BUNDLES
}

\author{
PETER BOUWKNEGT, KEITH HANNABUSS, AND VARGHESE MATHAI
}

\begin{abstract}
In this paper we study T-duality for principal torus bundles with H-flux. We identify a subset of fluxes which are T-dualizable, and compute both the dual torus bundle as well as the dual H-flux. We briefly discuss the generalized Gysin sequence behind this construction and provide examples both of non T-dualizable and of T-dualizable H-fluxes.
\end{abstract}

\section{INTRODUCTION}

T-duality is one of the most powerful tools in (super)string theory. It provides an equivalence between string theories which, in their low-energy field theory limit might superficially look very different, but are in fact the same in the sense that there exists a 1-1 correspondence between fields, states, etc. In particular, T-duality should relate the various D-branes in the theory, which is mathematically expressed as the fact that there should be an isomorphism between the relevant (twisted) K-theories (and their close cousin, (twisted) cohomology).

Locally, the T-duality transformation rules on the massless fields in string theory, known as the Buscher rules, have been known for quite some time [1]. Global issues, though, in particular in the background of NS H-flux, have remained obscure (see [2] for some early investigations).

In a recent paper, T-duality for principal circle bundles $\pi: E \rightarrow M$ (i.e. circle bundles with a free circle action), in the background of $\mathrm{H}$-flux $[H] \in H^{3}(E, \mathbb{Z})$ was examined [3, 4]. Such bundles are classified by their first Chern class $c_{1}(E) \in H^{2}(M, \mathbb{Z})$, and it was shown that T-duality interchanges the fibrewise integral of the H-flux with the first Chern class. I.e. $(E, H)$ and its T-dual $(\widehat{E}, \widehat{H})$ are related by

$$
c_{1}(E)=\int_{\widehat{\mathbb{T}}} \widehat{H}, \quad c_{1}(\widehat{E})=\int_{\mathbb{T}} H
$$

as can easily be argued from the Gysin sequences of the bundles $E$ and $\widehat{E}$. In addition, the isomorphisms between the twisted cohomologies and twisted K-theories of $(E, H)$ and its T-dual $(\widehat{E}, \widehat{H})$ were explicitly constructed.

In the present paper we will generalize these results to principal torus bundles, and identify a subset of T-dualizable H-fluxes which admit T-duals in a completely analogous way to the circle bundle case. We certainly do not want to claim that T-duals do not exist if one goes outside of this restrictive class, in fact it is well-known that the torus action need not be free, i.e. the torus action may have fixed points. Many examples of T-duals in this more general set-up, in particular in the context of mirror symmetry (see, e.g., [5] for the original idea, and [6] for most recent developments), have been constructed, but as far as we know no complete picture is known

$\mathrm{PB}$ and VM are financially supported by the Australian Research Council, and KH would like to thank the Department of Pure Mathematics at the University of Adelaide for hospitality during the course of this project. 
in the presence of H-flux. The purpose of this paper was to identify a class of torus bundles and H-fluxes which admit T-duals in the same class, and lead to isomorphisms in twisted cohomology and twisted K-theory, in a manner which generalizes the case of circle bundles. In particular we were aiming for a (generalized) Gysin sequence which relates the cohomologies of the torus-bundle $(E, H)$ and its T-dual $(\widehat{E}, \widehat{H})$.

The restriction to principal torus bundles is a natural one, physically it corresponds to the situation where momentum along the torus directions is conserved. In the case of circles bundles, all orientable circle bundles are in fact principal circle bundles. An example of a non-principal (and therefore non-orientable) circle bundle over the circle is the Klein bottle for which the analysis in [3] does not apply. For torus bundles, though, the situation is completely different. There are many more torus bundles than principal torus bundles (principal torus bundles over the torus have been classified in [7]). Moreover, even in the case of principal torus bundles not all H-fluxes admit a straightforward T-dual. There is a subset of H-fluxes, essentially those closed 3-forms which only have one 'leg' in the torus direction, which do however admit a T-dual, which is again a principal torus bundle with an H-flux in the same 'T-dualizable' subset.

The paper is organized as follows. In Section 2 we discuss the subset of T-dualizable H-fluxes on principal torus bundles, and give an explicit characterization of both the dual torus bundle as well as the dual H-flux. We also show how this would fit into a generalized Gysin sequence. In Section 3] we discuss some examples of both T-dualizable torus bundles (such as the group manifold) and non T-dualizable torus bundles, and discuss the complications which arise for non principal torus bundles.

For reasons of clarity, we restrict the discussion in this paper to T-duality aspects pertaining to the image of integral cohomology classes in de-Rham cohomology, i.e. the cohomology of differential forms with integral periods. The full result, as well as further details on the results in this paper, will be dealt with in a companion paper [8].

\section{T-DUALITY FOR PRINCIPAL TORUS BUNDLES}

2.1. $T$-dualizable H-fluxes. Let us denote the circle by $\mathbb{T}$, the $n$-torus by $\mathbb{T}^{n}$, or $\mathrm{T}$ for short. $\mathrm{T}$ can be considered as an (abelian) Lie group, and we let $\mathfrak{t}$ denote the Lie algebra of $\mathbf{T}$, and $\hat{\mathfrak{t}}$ the dual Lie algebra.

Now let $\pi: E \rightarrow M$ be a principal T-bundle. The action of $\mathrm{T}$ on $E$ associates to each element $X \in \mathfrak{t}$ a vector field on $E$ which, by abuse of notation, we will also denote as $X$. We will denote the Lie derivative and contraction with respect to the vector field $X$ as $\mathcal{L}_{X}$ and $\imath_{X}$, respectively.

Let $\Omega^{k}(N)$ and $\Omega^{k}(N, \mathfrak{t})$ denote the set of $k$-forms, and $\mathfrak{t}$-valued $k$-forms, on $N$, respectively, and let $H^{k}(N)$ and $H^{k}(N, \mathfrak{t})$ be the associated de-Rham cohomology groups of differential forms with integral periods. [In the rest of the paper the integrality conditions on closed forms will not be explicitly stated.] A form $\omega \in \Omega^{k}(E)$ is called basic if $\omega$ is the pull-back of a form on the base manifold $M$. This is equivalent to the requirement that $\mathcal{L}_{X} \omega=\imath_{X} \omega=0$ for all $X \in \mathfrak{t}$. An H-flux on $E$ is, by definition, a closed, integral, 3-form $H \in \Omega^{3}(E)$, i.e. it determines a class $[H] \in H^{3}(E)$. 
Definition 2.1. An H-flux $H$ is called T-dualizable when there exists a closed, $\hat{\mathfrak{t}}$-valued, 2 -form $\widehat{F}$ on $M$ such that the pair $(H, \widehat{F})$ satisfies

$$
d H=0, \quad \imath_{X} H=\pi^{*} \widehat{F}(X),
$$

for all $X \in \mathfrak{t}$, where $\widehat{F}(X) \in \Omega^{2}(M)$ denotes the dual pairing of $\widehat{F} \in \Omega^{2}(M, \hat{\mathfrak{t}})$ with $X \in \mathfrak{t}$.

Remark. More generally, one may define a T-dualizable flux as a pair $(H, \widehat{F})$ such that the relations (2.1) hold at the level of cohomology only. As this would unnecessarily complicate the discussion below, we will simply assume that representatives $(H, \widehat{F})$ have been chosen such that (2.1) holds at the level of forms.

Note, that the closed 2-form $\widehat{F}$ on $M$, i.e. $[\widehat{F}] \in H^{2}(M, \hat{\mathfrak{t}})$, determines a principal $\widehat{\mathrm{T}}$-bundle $\hat{\pi}: \widehat{E} \rightarrow M$, which we will refer to as the T-dual torus bundle. In fact, to be precise, $[\widehat{F}]$ only determines $\widehat{E}$ up to torsion. To determine the torsion part of $\widehat{E}$ we need to work with integer cohomology classes. As the purpose of this paper is to explain the main ideas behind the construction, we will simply accept the fact that if we were to work with the 'appropriate' cohomology theory the T-dual bundle $\widehat{E}$ would be uniquely determined (up to isomorphism). As far as providing the isomorphism of the twisted cohomology of $E$ with that of $\widehat{E}$ the torsion part of $\widehat{E}$ is irrelevant.

From Definition 2.1 it follows that all T-dualizable H-fluxes $(H, \widehat{F})$ necessarily satisfy $\mathcal{L}_{X} H=$ $0=\mathcal{L}_{X} \widehat{F}$ for all $X \in \mathfrak{t}$. In fact, it is well-known that every closed form on $E$ is cohomologous to a closed form $\omega$ on $E$ that satisfies $\mathcal{L}_{X} \omega=0$. So, without loss of generality, we may assume that all forms in question are invariant. On this subspace the de-Rham differential $d$ anti-commutes with the contraction $\imath_{X}$, since $\mathcal{L}_{X}=\left\{d, \imath_{X}\right\}$, so, after defining a locally defined 1-form $\widehat{A} \in \Omega_{\text {loc }}^{1}(E, \mathfrak{t})$ such that $\pi^{*} \widehat{F}=d \widehat{A}$, we may interpret the conditions (2.1) on the pair $(H, \widehat{A})$ as defining some sort of Deligne cohomology class in a double complex, except for the fact that $\widehat{A}$ is only locally defined on $E$. One might be tempted to think that the pull-back $\pi^{*} \widehat{F}$ is exact on $E$, and that therefore $\widehat{A}$ is globally defined on $E$, but this is incorrect. In fact, the set-up is precisely such that $\hat{\pi}^{*} \widehat{F}$ is exact on the T-dual bundle $\widehat{E}$, and that the various locally defined $\widehat{A}$ patch together to form a globally defined connection 1 -form, with values in $\hat{\mathfrak{t}}$, on $\widehat{E}$, such that $\hat{\pi}^{*} \widehat{F}=d \widehat{A}$. Without loss of generality we assume that $\widehat{A} \in \Omega^{1}(\widehat{E}, \hat{\mathfrak{t}})$ is normalized such that

$$
\imath_{X} \widehat{A}=X,
$$

for all $X \in \hat{\mathfrak{t}}$.

Remark. Finally, we remark that the conditions (2.1), written in terms of $(H, \widehat{A})$, are remarkably similar to the conditions defining an equivariant cohomology class, i.e., a class in $H_{\top}^{3}(E)$. Equivariant cohomology works with differential forms which are also polynomials on the Lie algebra $\mathfrak{t}$ of the group $\mathrm{T}$, where the total degree of a homogeneous form $\alpha$ is defined as the sum of the form degree of $\alpha$ plus twice the polynomial degree. The equivariant differential $d_{\mathrm{T}}$ is defined as

$$
d_{\top} \alpha(X)=d \alpha(X)-\imath_{X} \alpha(X) .
$$


Written in terms of $\alpha=H+\widehat{A}$, the condition that $\alpha$ is closed under $d_{\text {T }}$ gives, after collecting the terms of the same form degree,

$$
d H=0, \quad \imath_{X} H=d \widehat{A}(X), \quad \imath_{X} \widehat{A}(X)=0 .
$$

The first two equations correspond to (2.1), while the latter is a normalization condition on $\widehat{A}$. The difference between Eqn. (2.1) and equivariant cohomology is, though, that $\widehat{A}$ is only defined locally. In fact, since $\mathrm{T}$ acts freely on $E$, one would have $H_{\mathrm{T}}(E) \cong H(M)$, which makes equivariant cohomology not particularly useful in this case.

2.2. The T-dual H-flux. In Sect. 2.1 we have seen how, given a T-bundle $\pi: E \rightarrow M$, and a T-dualizable H-flux $(H, \widehat{F})$, we have defined a T-dual $\widehat{T}$-bundle $\hat{\pi}: \widehat{E} \rightarrow M$, with connection $\widehat{A} \in \Omega^{1}(\widehat{E}, \hat{\mathfrak{t}})$, such that $\hat{\pi}^{*} \widehat{F}=d \widehat{A}$, and normalized according to Eqn. (2.2). The goal of this section is to define the T-dual H-flux. It will turn out that the T-dual $\widehat{H}$ is a T-dualizable H-flux on $\widehat{E}$, as one would hope.

As in [4] we could proceed to find $\widehat{H}$, up to a basic form, from a generalized Gysin sequence (see Sect. 2.3). Here we proceed by simply defining $\widehat{H}$ and show it has the required properties.

Let $A$ be a connection 1 -form on $E$, that is $A \in \Omega^{2}(E, \mathfrak{t})$ such that its curvature $F=d A$ is (the pull-back of) a closed 2-form on $M$. We normalize $A$ such that $\imath_{X} A=X$. These together imply that $\mathcal{L}_{X} A=0$. Since the T-dual connection and $\mathrm{H}$-flux live on a different space as the original connection and H-flux, in order to compare them we need to pull all of these forms back to a common space, known as a correspondence space. The correspondence space, in this case, is the fibred product of $E$ and $\widehat{E}$, i.e.

$$
E \times{ }_{M} \widehat{E}=\{(x, \hat{x}) \in E \times \widehat{E} \mid \pi(x)=\hat{\pi}(\hat{x})\} .
$$

The projection $p: E \times_{M} \widehat{E} \rightarrow E$ is given as the composition

$$
E \times \times_{M} \widehat{E} \stackrel{1 \otimes \hat{\pi}}{\longrightarrow} E \times_{M} M \stackrel{\cong}{\longrightarrow} E
$$

and defines $E \times_{M} \widehat{E}$ as a principal $\widehat{T}$-bundle over $E$. Similarly for $\hat{p}$. This shows we have a commutative diagram of torus bundles

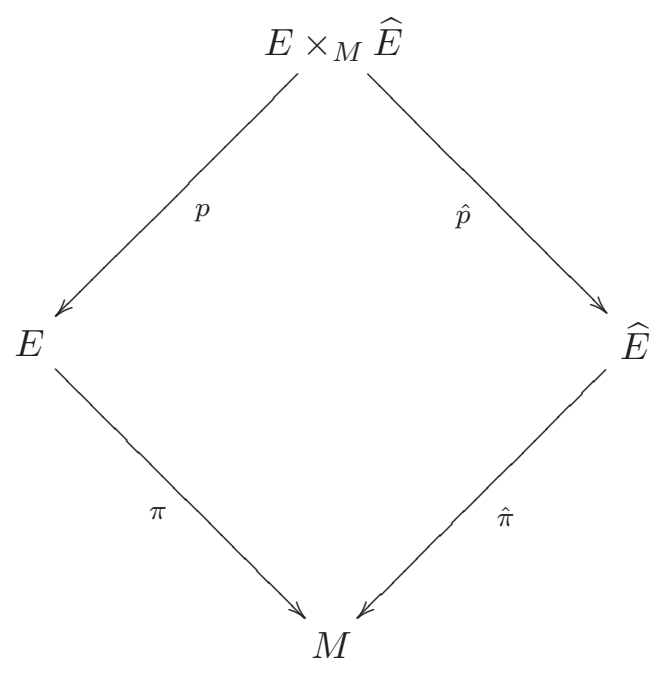


In the remainder of this section all forms are pulled back to the space on which the equation makes sense, but for notational simplicity we will omit the pull-backs from the equations.

First of all, consider the difference $\Omega=A \sqcap d \widehat{A}-H$, where we define the $\sqcap$ as the wedge between forms followed by the canonical pairing between $\hat{\mathfrak{t}}$ and $\mathfrak{t}$. A priori, $\Omega$ is a form on $E$, but we will show that $\Omega$ is actually a basic 3 -form. Obviously we have $\mathcal{L}_{X} \Omega=0$, and a little calculation gives

$$
\imath_{X} \Omega=d \widehat{A}(X)-\imath_{X} H=0,
$$

which together imply that $\Omega$ is basic. In other words, this implies that our T-dualizable H-flux $H$ can be written as

$$
H=A \sqcap d \widehat{A}-\Omega,
$$

for some $\Omega \in \Omega^{3}(M)$. Note that, in particular,

$$
d A \sqcap d \widehat{A}=d \Omega,
$$

hence $[F \sqcap \widehat{F}]=0$ in $H^{4}(M)$.

We now define the T-dual H-flux on $\widehat{E}$ to be

$$
\widehat{H}=d A \sqcap \widehat{A}-\Omega .
$$

Then, a simple calculation shows that $\widehat{H}$ is a closed, $\widehat{\mathrm{T}}$-invariant, 3 -form on $\widehat{E}$. Moreover it is T-dualizable, since

$$
\imath_{\hat{X}} \widehat{H}=d A(\widehat{X})=F(\widehat{X}) .
$$

Finally, observe that on the correspondence space $E \times_{M} \widehat{E}$, the difference $H-\widehat{H}$ is exact $^{1}$, since

$$
H-\widehat{H}=A \sqcap d \widehat{A}-d A \sqcap \widehat{A}=-d(A \sqcap \widehat{A}) .
$$

This, in particular implies that $A \sqcap \widehat{A}$ intertwines between the twisted cohomologies (and twisted K-theories) of $(E, H)$ and $(\widehat{E}, \widehat{H})$. The explicit isomorphism between the $\mathbb{Z}_{2}$-graded twisted cohomologies of $E$ and $\widehat{E}$, i.e. $H^{\bar{i}}(E,[H]) \cong H^{\overline{i+n}}(\widehat{E},[\widehat{H}])$ where $n$ is the rank of the torus and $\bar{i}=i$ $\bmod 2$, is a straightforward generalization of the result in [3], namely that the Buscher rules on the $\mathrm{RR}$ fields can be encoded in the formula,

$$
G \mapsto \widehat{G}=\int_{\top} e^{A \sqcap \widehat{A}} G .
$$

This reduces to the familiar formula for the Buscher rules in [1, 10], both locally, and globally when the flux $H=d B$ is exact. A similar generalization exist for the isomorphism between the respective twisted K-theories, $K^{\bar{i}}(E,[H]) \cong K^{\overline{i+n}}(\widehat{E},[\widehat{H}])$, and the two isomorphisms are compatible in the sense that the Riemann-Roch theorem holds in this case as well (see [8] for more details).

Remark. It is well-known that an $n$-torus, for $n>1$, admits a group of T-dualities, namely $S O(n, n ; \mathbb{Z})$. Additional T-dualities can be recovered in the formalism above by taking noncanonical pairings between $\mathfrak{t} \cong \mathbb{R}^{n}$ and $\hat{\mathfrak{t}} \cong \mathbb{R}^{n}$.

\footnotetext{
${ }^{1} \mathrm{~A}$ similar conclusion was reached in [9], Sect. 4 .
} 
2.3. The generalized Gysin sequence. In this section we will briefly indicate how the above construction would nicely fit in the framework of a generalized Gysin sequence. In fact, the existence of this Gysin sequence motivated our restriction to principal torus bundles and our definition of T-dualizable H-fluxes. The Gysin sequence we are about to discuss is the de-Rham analogue of the Gysin sequence in [11].

In general, a torus bundle, or any fibre bundle, gives rise to a spectral sequence (the so-called Leray spectral sequence) computing the cohomology of the bundle space from the cohomology of the base space and the fibre. In the case of sphere bundles this spectral sequence collapses into a long exact sequence, the Gysin sequence, in cohomology (see, e.g., [12]), but this is not the case for torus bundles. But even if it did, with the application to T-duality in mind we are interested in relations between cohomology groups involving the cohomology group that classifies (a subclass of) torus bundles. Principal $n$-torus bundles over a base space $M$ are classified by the sheaf cohomology group $H^{1}(M, \underline{\mathbf{T}}) \cong H^{2}\left(M, \mathbb{Z}^{n}\right)$, whose image in de-Rham cohomology can be identified with $H^{2}(M, \mathfrak{t})$. Thus, for a given torus bundle $\pi: E \rightarrow M$ we are looking for a long exact sequence relating the cohomologies $H^{k}(E)$ and $H^{k}(M, \mathfrak{t})$. The missing ingredient is a third cohomology group $H_{\mathrm{R} W}^{k}(E, \mathfrak{t})$. It was introduced, in the sheaf language, by Raeburn and Williams in [13], where it was refered to as t-equivariant cohomology. However, while it is closely related to the conventional equivariant cohomology corresponding to the T-action on $E$, it is in general not the same (cf. the discussion in Sect. 2.2). To avoid confusion we will refer to it as the RW-cohomology, and use the notation $H_{\mathrm{R} W}^{k}(E, \mathfrak{t})$. Its definition is generalizing Definition 2.1

An element in $H_{\mathrm{R} W}^{k}(E, \mathfrak{t})$ is a pair $(H, \widehat{F})$, with $H \in \Omega^{k}(E)$ and $\widehat{F} \in \Omega^{k-1}(M, \hat{\mathfrak{t}})$, such that the following conditions are satisfied for all $X \in \mathfrak{t}$

$$
d H=0, \quad \imath_{X} H=\pi^{*} \widehat{F}(X), \quad d \widehat{F}=0
$$

The RW-cohomology is the set of such pairs modulo pairs of the form $(H, \widehat{F})=(d B, d C)$, where $B \in \Omega^{2}(E)$ and $C \in \Omega^{1}(M, \hat{\mathfrak{t}})$, such that $\imath_{X} B=\pi^{*} C(X)$, for all $X \in \mathfrak{t}$.

As an aside, let us remark that the Čech analogue of $H_{\mathrm{R} W}^{k}(E, \mathfrak{t})$ is the sheaf cohomology group $H_{\mathrm{RW}}^{k-1}(E, \mathfrak{t}, \underline{\mathbb{T}})$, and that $H_{\mathrm{R} W}^{1}(E, \mathfrak{t}, \mathbb{\mathbb { T }})$ is in 1-1 correspondence with the isomorphism classes of T-equivariant line bundles over $E$ that are locally trivial over $M=E / \mathrm{T}\left[11\right.$, while $H_{\mathrm{R} W}^{2}(E, \mathfrak{t}, \mathbb{T})$ is in 1-1 correspondence with the stable isomorphism classes of T-equivariant bundle gerbes $L \rightarrow E^{[2]}$ that are locally trivial over $E^{[2]} / \mathrm{T}[8]$.

Now, given a principal T-bundle $\pi: E \rightarrow M$ characterized by a curvature $F$, i.e. $[F] \in H^{2}(M, \mathfrak{t})$, we expect to derive a long exact sequence in cohomology (for $k \geq 1$ ), the so-called generalized Gysin sequence, given as in [11, 8] by,

$$
\ldots \longrightarrow H^{k}(M) \stackrel{p^{*}}{\longrightarrow} H_{\mathrm{R} W}^{k}(E, \mathfrak{t}) \stackrel{b}{\longrightarrow} H^{k-1}(M, \hat{\mathfrak{t}}) \stackrel{\sqcap F}{\longrightarrow} H^{k+1}(M) \longrightarrow \ldots
$$


where the various maps are explicitly given $b y^{2}$

$$
\begin{array}{cr}
p^{*}: H^{k}(M) \rightarrow H_{\mathrm{R} W}^{k}(E, \mathfrak{t}), & H \mapsto\left(\pi^{*} H, 0\right), \\
b: H_{\mathrm{R} W}^{k}(E, \mathfrak{t}) \rightarrow H^{k-1}(M, \hat{\mathfrak{t}}), & (H, \widehat{F}) \mapsto \widehat{F}, \\
\sqcap F: H^{k-1}(M, \hat{\mathfrak{t}}) \rightarrow H^{k+1}(M), & \widehat{F} \mapsto \widehat{F} \sqcap F .
\end{array}
$$

In the last definition, we remind the reader that $\widehat{F} \sqcap F$ stands for taking both the wedge product of the $\hat{\mathfrak{t}}$-valued $(k-1)$-form $\widehat{F}$ with the $\mathfrak{t}$-valued 2 -form $F$, as well as the canonical pairing between $\hat{\mathfrak{t}}$ and $\mathfrak{t}$, to produce an $\mathbb{R}$-valued $(k+1)$-form.

The results and computations in Sects. 2.1] and 2.2 can easily be interpreted as 'diagram chasing' in this generalized Gysin sequence (the $k=3$ segment, in particular) in an analogous manner to the discussion in 3]. Finally, we remark that the circle bundle case is obtained from the more general case above by observing that $H_{\mathrm{R} W}^{k}(E, \mathfrak{t}) \cong H^{k}(E)$, for $k \geq 1$, if $E$ is a principal circle bundle (cf. [11]).

\section{EXAmples}

3.1. The trivial $\mathbb{T}^{2}$-bundle over $\mathbb{T}$. There are no nontrivial principal $\mathbb{T}^{2}$-bundles over $\mathbb{T}$ as $H^{1}\left(\mathbb{T}, \underline{\mathbb{T}}^{2}\right) \cong H^{2}\left(\mathbb{T}, \mathbb{Z}^{2}\right)=0$. In particular, the nilmanifold ('twisted 3 -torus') that enters in many physically interesting examples, is a nontrivial $\mathbb{T}^{2}$-bundle over $\mathbb{T}$, but it is not principal.

A simple example of a principal torus bundle with non T-dualizable $\mathrm{H}$-flux is provided by $\mathbb{T}^{3}$, considered as the trivial $\mathbb{T}^{2}$-bundle over $\mathbb{T}$, with $H$ given by $k$ times the volume form on $\mathbb{T}^{3}$. In this case $\imath_{X} H$, with $X \in \mathfrak{t}$, is not a basic 2 -form for obvious reasons, hence $H$ is non T-dualizable in the sense of Definition 2.1

It is illuminating to work out explicitly what happens in this case, as naively one might T-dualize one circle at a time by applying the Buscher rules (see also the discussion in [3]). Explicitly, in terms of Cartesian coordinates $(x, y, z) \sim(x+1, y, z) \sim(x, y+1, z) \sim(x, y, z+1)$ we have a metric $g$ and H-flux $H$ given by

$$
g=d x^{2}+d y^{2}+d z^{2}, \quad H=k d x \wedge d y \wedge d z .
$$

After choosing a local gauge $H=d B$, with $B=k x d y \wedge d z$, application of the Buscher rules [1] to the circle defined by $z$ yields

$$
\widehat{g}=d x^{2}+d y^{2}+(d \hat{z}+k x d y)^{2}, \quad \widehat{H}=0,
$$

which can be interpreted as a metric on the nilmanifold defined by $(x, y, \hat{z}) \sim(x+1, y, \hat{z}-k y) \sim$ $(x, y+1, \hat{z}) \sim(x, y, \hat{z}+1)$. We would now like to apply the Buscher rules to the circle defined by $y$, but it is clear from the identifications that there is no corresponding circle action - which is related to the fact that the nilmanifold is not a principal $\mathbb{T}^{2}$-bundle. A naive application of the Buscher rules gives results which are suspicious, as the transformed metric does not appear to be a metric on any torus bundle [14. The conclusion is that if one tries to T-dualize a principal torus bundle with a non T-dualizable H-flux, in the sense of Def. 2.1] it will certainly take us out of the realm

\footnotetext{
${ }^{2}$ We write the maps as given on representatives of the various cohomology groups for notational simplicity.
} 
of principal torus bundles or, perhaps, there is even a genuine obstruction for such a T-duality. Similarly, T-duality for non principal torus bundles is not straightforward, or perhaps problematic, for the same reasons.

3.2. The group manifold. For an example of a T-dualizable flux consider a simple, compact Lie group G. Let $\mathrm{T}$ denote its maximal torus. We can consider $\mathrm{G}$ as a principal T-bundle over the flag manifold G/T.

Let $\left[H_{0}\right]$ denote the generator of $H^{3}(\mathrm{G}, \mathbb{Z}) \cong \mathbb{Z}$, and let $H_{0}$ be a de-Rham representative of $\left[H_{0}\right]$. Explicitly, if $\operatorname{Tr}$ denotes a properly normalized trace on the Lie algebra $\mathfrak{g}$ of $\mathrm{G}$, we may take

$$
H_{0}=\operatorname{Tr}(\Theta \wedge \Theta \wedge \Theta)
$$

where $\Theta=g^{-1} d g$ denotes the left-invariant Maurer-Cartan 1-form on G.

Now consider the H-flux $H=k H_{0}$, for some positive integer $k$, and let $X \in \mathfrak{t}$. We have

$$
\imath_{X} H=k \operatorname{Tr}(X \Theta \wedge \Theta)=-k d(\operatorname{Tr}(X \Theta))
$$

where, in the second step, we have used the Maurer-Cartan equation $d \Theta+\Theta \wedge \Theta=0$. First, consider $\widehat{F} \in \Omega^{2}(\mathrm{G}, \hat{\mathfrak{t}})$ defined by $\widehat{F}(X)=k \operatorname{Tr}(X \Theta \wedge \Theta)$. Since, for any $Y \in \mathfrak{t}$ we have $\mathcal{L}_{Y} H=0$, the same

holds for $\widehat{F}$, i.e. $\mathcal{L}_{Y} \widehat{F}=0$. Moreover, for all $X, Y \in \mathfrak{t}$, we have $\imath_{Y} \widehat{F}(X)=k \operatorname{Tr}([X, Y] \Theta)=0$ hence $\imath_{Y} \widehat{F}=0$. This shows that $\widehat{F}$ is a basic, closed, $\hat{\mathfrak{t}}$-valued 2-form, i.e. $[\widehat{F}] \in H^{2}(\mathrm{G} / \mathrm{T}, \hat{\mathfrak{t}})$. Moreover, the second equality shows that we can write $\widehat{F}=d \widehat{A}$, with $\widehat{A} \in \Omega^{1}(\widehat{\mathrm{G}}, \hat{\mathfrak{t}})$ defined by $\widehat{A}(X)=-k \operatorname{Tr}(X \Theta)$. In particular this shows that all H-fluxes on a group manifold are T-dualizable with respect to the maximal torus $\mathrm{T}$ (or, with respect to any subtorus, for that matter).

Note that $\widehat{A}$ is not a basic form, but rather a globally defined connection on a T-dual $\widehat{T}$-bundle $\widehat{\mathrm{G}}$. It is not hard to see that, in fact, $\widehat{\mathrm{G}}=\mathrm{G} /\left(\mathbb{Z}_{k}\right)^{r}$, where $r$ is the rank of $\mathrm{G}$ and $\left(\mathbb{Z}_{k}\right)^{r} \subset \mathrm{T}=\mathbb{T}^{r}=$ $\mathbb{T} \times \mathbb{T} \times \ldots \times \mathbb{T}$ is the subgroup of $T$ such that each $\mathbb{Z}_{k} \subset \mathbb{T}$ with generator $\exp (2 \pi i / k)$. In the case of $\mathrm{G}=S U(2)$, the T-dual manifold $\mathrm{G} / \mathbb{Z}_{k} \cong S^{3} / \mathbb{Z}_{k}$ is the Lens space $L(1, k)$, and we reproduce the result of $[15,3$.

The example of the group manifold can be generalized to more general principal T-bundles over the flag manifold $G / T$, and leads to a whole web of dualities with corresponding isomorphisms between the respective twisted cohomologies and K-theories.

\section{REFERENCES}

[1] T. Buscher, A symmetry of the string background field equations, Phys. Lett. B194 (1987) 59-62; ibid., Path integral derivation of quantum duality in nonlinear sigma models, Phys. Lett. B201 (1988) 466-472.

[2] E. Álvarez, L. Álvarez-Gaumé, J.L.F. Barbón and Y. Lozano, Some global aspects of duality in string theory, Nucl. Phys. B415 (1994) 71-100, arXiv:hep-th/9309039.

[3] P. Bouwknegt, J. Evslin and V. Mathai, T-duality: Topology change from H-flux, Comm. Math. Phys., to be published, arXiv:hep-th/0306062.

[4] P. Bouwknegt, J. Evslin and V. Mathai, On the topology and H-flux of T-dual manifolds, arXiv:hep-th/0312052.

[5] A. Strominger, S.-T. Yau and E. Zaslow, Mirror symmetry is T-duality, Nucl. Phys. B479 (1996) 243-259, arXiv:hep-th/9606040.

[6] R. Donagi and T. Pantev, Torus fibrations, gerbes and duality, arXiv:math.AG/0306213. 
[7] R.S. Palais and T.E. Stewart, Torus bundles over a torus, Proc. Amer. Math. Soc. 12 (1961) 26-29.

[8] P. Bouwknegt, K. Hannabuss and V. Mathai, in preparation.

[9] O. Alvarez, Target space duality I: General Theory, Nucl. Phys. B584 (2000) 659-681, arXiv:hep-th/0003177.

[10] K. Hori, D-branes, T-duality, and index theory, Adv. Theor. Math. Phys. 3 (1999) 281-342, arXiv:hep-th/9902102.

[11] J. Packer, I. Raeburn and D.P. Williams The equivariant Brauer group of principal bundles, J. Operator Theory 36 (1996) 73-105.

[12] R. Bott and L.W Tu, Differential forms in algebraic topology, (Springer Verlag, New York, 1982).

[13] I. Raeburn and D.P. Williams, Dixmier-Douady classes of dynamical systems and crossed products, Can. J. Math. 45 (1993) 1032-1066.

[14] S. Kachru, M. Schulz, P. Tripathy and S. Trivedi, New supersymmetric string compactifications, J. High Energy Phys. 03 (2003) 061, arXiv:hep-th/0211182.

[15] J. Maldacena, G. Moore and N. Seiberg, D-brane charges in five-brane backgrounds, J. High Energy Phys. 10 (2001) 005, arXiv:hep-th/0108152.

(Peter Bouwknegt) Department of Physics and Mathematical Physics, and Department of Pure Mathematics, University of Adelaide, Adelaide, SA 5005, Australia

E-mail address: pbouwkne@physics.adelaide.edu.au, pbouwkne@maths.adelaide.edu.au

(Keith Hannabuss) Department of Mathematics, University of Oxford, England

E-mail address: kch@balliol.oxford.ac.uk

(Varghese Mathai) Department of Pure Mathematics, University of Adelaide, Adelaide, SA 5005, Australia

E-mail address: vmathai@maths.adelaide.edu.au 